\title{
White-Black Differences in Cancer Incidence, Stage at Diagnosis, and Survival Among Older Adults
}

\author{
Jessica L. Krok-Schoen, $\mathrm{PhD}^{1,2}$, James L. Fisher, $\mathrm{PhD}^{3}$, Ryan D. Baltic, $\mathbf{M P H}^{2}$, and Electra \\ D. Paskett, $\mathrm{PhD}^{2,4,5}$ \\ ${ }^{1}$ Division of Medical Dietetics and Health Sciences, School of Health and Rehabilitation Sciences, \\ College of Medicine, The Ohio State University, Columbus, OH, USA \\ ${ }^{2}$ Comprehensive Cancer Center, The Ohio State University, Columbus, $\mathrm{OH}, \mathrm{USA}$ \\ ${ }^{3}$ Arthur G. James Cancer Hospital and Richard J. Solove Research Institute, Columbus, OH, USA \\ ${ }^{4}$ Division of Epidemiology, College of Public Health, The Ohio State University, Columbus, $\mathrm{OH}$, \\ USA \\ ${ }^{5}$ Division of Cancer Prevention and Control, Department of Internal Medicine, College of \\ Medicine, The Ohio State University, Columbus, OH, USA
}

\section{Abstract}

Objective-To identify potential White-Black differences in cancer incidence rates, stage at diagnosis, and relative survival probabilities among older adults using Surveillance, Epidemiology, and End Results (SEER) data.

Method-Differences in cancer incidence, stage at diagnosis, and 5-year relative survival probability were examined for cases diagnosed within the most recent 5-year period and over time for cases diagnosed from 1973 to 2013 (incidence only) for older White and Black adults.

Results-Among adults aged 65 to 74,75 to 84 , and 85 years and older, Black adults had higher cancer incidence rates per 100,000 than White males from 1973 to 2013, respectively. Late stage and unstaged cancers were more common among Black adults in each of the three age groups compared with Whites. Five-year relative survival probability for all invasive cancers combined was higher for Whites than Blacks in each of the three age groups.

Discussion-Continued efforts are needed to reduce racial disparities in cancer incidence and mortality among older adults.

\section{Keywords}

cancer disparities; cancer incidence; cancer survivorship; SEER program

Reprints and permissions: sagepub.com/journalsPermissions.nav

Corresponding Author: Jessica L. Krok-Schoen, Division of Medical Dietetics and Health Sciences, School of Health and Rehabilitation Sciences, College of Medicine, The Ohio State University, 306 Atwell Hall, 453 W. 10th Avenue, Columbus, OH 43201, USA. Jessica.krok@ osumc.edu.

Declaration of Conflicting Interests

The authors declared no potential conflicts of interest with respect to the research, authorship, and/or publication of this article. 
Increased life expectancy and advances in cancer screening have resulted in an increasing

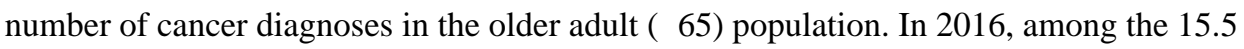
million cancer survivors in the United States, $62 \%$ were older adults (Bluethmann, Mariotto, \& Rowland, 2016). By 2040, cancer prevalence is expected to increase to 26.1 million and older adults will account for $73 \%$ of the survivors (Bluethmann et al., 2016). In addition to the overall growth of the older cancer patient population, the population will also become more racially diverse. In the United States, racial minority populations are expected to increase from 83 million in 2000 to 157 million in 2030 and experience more than a 100\% increase in cancer incidence by 2030 (Smith, Smith, Hurria, Hortobagyi, \& Buchholz, 2009). These demographic and clinical changes will undeniably have a significant impact on cancer prevention, early detection, treatment, and survivorship (McKoy, Samaras, \& Bennett, 2009).

The changing U.S. demographics emphasize the urgency of awareness and elimination of cancer disparities among older adults. As seen in the pathways (Diez Roux, 2013; Shanahan $\&$ Hofer, 2005) and biopsychosocial models used by Freeman and Chu (2005) and Warnecke et al. (2008), the determinants of health disparities can range from personal (e.g., race, socioeconomic status [SES]) to societal (e.g., health policy, discrimination) and are multifactorial, dynamic, and interrelated. These multiple mechanisms coupled with a lifetime of inequity can alter gene expression and may manifest in the form of disease, decades after exposure (Braveman \& Gottlieb, 2014).

One factor, race, has long been known as a social determinant of health status (Wallace, 2015; Williams \& Mohammed, 2009). Older Blacks have consistently more major health problems and higher functional limitations as compared with non-Hispanic Whites (Smedley, Stith, \& Nelson, 2002; Wallace, 2015). Previous studies have identified continued White and Black differences across the cancer spectrum from prevention through screening, treatment, and survivorship (Siegel, Miller, \& Jemal, 2016; Ward et al., 2004). Black adults in the United States experience overall higher cancer incidence, younger age at diagnosis, more frequent late-stage diagnoses, less aggressive treatments, and higher mortality than White adults (DeSantis et al., 2016; Gross, Smith, Wolf, \& Andersen, 2008; Siegel et al., 2016; Ward et al., 2004). For example, Ward and colleagues (2004) found that Black cancer patients were less likely to receive recommended surgery than White cancer patients, even if they had the same income and insurance levels.

Cancer disparities by age exist as well. Studies found that older adults were given less aggressive cancer treatment options compared with their younger counterparts, even if they had similar functional status (Foster, Salinas, Mansell, Williamson, \& Casebeer, 2010; Freedman, He, Winer, \& Keating, 2009; Jessup, Stewart, Greene, \& Minsky, 2005; Mahal et al., 2014; Zeng et al., 2015). For example, Foster and colleagues (2010) found that for stage IIA breast cancer (Eastern Cooperative Oncology Group [ECOG] score $=0$ ), 93\% of physicians recommended intensive adjuvant treatment for a patient aged 63 years, but only $66 \%$ said they would do so if the patient's age was 75 . When the factors of age and race are combined, older Black adults with cancer may experience "double jeopardy," compounded detrimental effects on health outcomes, compared with their White counterparts (Bellizzi et al., 2012). Despite a rapidly expanding population of minority older adults, few studies have 
specifically examined cancer disparities among older adult populations, even for the more common cancer types (DeSantis et al., 2016; Gross et al., 2008; Siegel et al., 2016).

This study sought to identify potential White-Black differences in cancer incidence rates, stage at diagnosis, and relative survival probabilities among older adults using data from the Surveillance, Epidemiology, and End Results (SEER) program of the National Cancer Institute. The hypothesis that there is a continuation of cancer disparities between Whites and Blacks in the 65 to 74,75 to 84 , and $\geq 85$ populations, as evidenced in several studies of younger cohorts, was evaluated in the present study. If confirmed, results concerning this hypothesis may assist in the allocation of resources to reduce cancer disparities among older adults.

\section{Method}

Cancer incidence, stage at diagnosis, and survival data collected by the 18 population-based registries of the National Cancer Institute's SEER database were used for these analyses. These registries capture data covering approximately $30 \%$ of the U.S. population (Howlader et al., 2015). All data were publicly available, de-identified, and determined to be exempt from Institutional Review Board review.

White and Black adults, regardless of ethnicity, aged 65 to 74,75 to 84 , and $\geq 85$ years, diagnosed with invasive colorectal, lung and bronchus, breast (females only), and prostate cancer were included in analyses relating to cancer incidence and relative survival probability. In situ cancers were also included in analyses concerning stage at diagnosis. Because it was not feasible to examine incidence, stage at diagnosis, and survival for all the 23 SEER groupings of cancer sites/types in the scope of this effort, the four most common cancers were examined; these represent more than half (52.7\%) of invasive cancers among older adults.

Using SEER*Stat statistical software (Version 8.3.2; National Cancer Institute), differences in cancer incidence, stage at diagnosis, tumor grade and size, and treatments of surgery and radiation were examined among older Whites and Black adults for cases diagnosed from 2009 through 2013, while changes in incidence over time were examined for cases diagnosed from 1973 through 2013. For comparisons of 2009-2013 data (incidence, stage at diagnosis, tumor grade and size, treatments), 18 SEER registries were used (SEER program, 2016b). For comparisons of trends in incidence rates from 1973 to 2013, data from the original nine SEER registries were used (SEER program, 2016a). For comparisons of relative survival probabilities (SEER program, 2016b), 18 SEER registries were used. All cancer incidence rates were age-adjusted using the 2000 U.S. standard population (19 age groups- Census P25-1130). For sex-specific cancers (prostate, female breast), rates were calculated using sex-specific denominators.

The SEER factor "derived SEER summary stage (2000)" was used to determine the proportion diagnosed late (regional and distant) stage, and the SEER factor "CS tumor size" was used to characterize tumor size. Proportions were used to ascertain potential differences in stage at diagnosis, tumor grade, and treatments of surgery and radiation. Differences in

J Aging Health. Author manuscript; available in PMC 2019 July 01. 
tumor size were characterized using the median values among those with known values.

Five-year relative survival probability for invasive colorectal, lung and bronchus, breast, and prostate cancers were examined for Whites and Blacks, regardless of ethnicity among 65- to 74-, 75- to 84-, and 285 -year-olds and diagnosed from 2006 to 2012. Relative survival refers to the ratio of the proportion of observed survivors in a cohort of cancer patients to the proportion of expected survivors in a comparable set of cancer-free individuals in a designated time period (in this case, 5 years; SEER program, 2017). It does not distinguish between patients who have no evidence of cancer and those who have relapsed or are still in treatment. Years selected above for incidence, stage at diagnosis, and relative survival probability were chosen because they are the most recent years available and to be consistent with years included in summary statistics reported in the Cancer Statistics Review (Howlader et al., 2015).

\section{Results}

There were 997,454 White and Black men and women aged $\ 65$ years diagnosed with invasive cancer in the 18 SEER registries from 2009 to 2013. Of the 997,454 older White and Black adults with cancer, 525,604 were diagnosed with one of the four cancer sites of interest: 98,034 (18.7\%) individuals were diagnosed with colorectal, 165,446 (31.5\%) with lung and bronchus, 119,723 (22.8\%) with breast (women only), and 142,401 (27.1\%) with prostate cancer within this population. Of these 525,604 cases, 468,400 (89.1\%) were White and 57,204 (10.9\%) were Black.

\section{Incidence}

Figure 1 shows the overall incidence trends for all common cancer types combined among older White and Black adults by age group. Older Black adults, particularly those in the 65 to 74 and 75 to 84 age groups, had higher overall incidence of combined cancers (colorectal, lung and bronchus, breast, and prostate) over time. Figure 2a to $2 \mathrm{~d}$ shows the incidence trends of invasive colorectal, lung and bronchus, breast, and prostate cancer among White and Black adults aged 65 to 74,75 to 84, and $\geq 85$ years from 1973 to 2013, respectively.

Colorectal cancer-Overall, colorectal cancer incidence declined over time among older White and Black adults. Specifically, colorectal cancer incidence rates among Black adults aged 65 to 74 and 75 to 84 years, in general, exceeded those of Whites since 1987 and 1997, respectively.

Lung and bronchus cancer-In general, lung and bronchus cancer incidence increased over time among older White and Black adults aged 75 to 84 and $\geq 85$ years. From 1973 to 2013, Blacks aged 65 to 74 years had higher lung and bronchus cancer incidence rates than White adults. The clear White- Black difference in lung and bronchus cancer incidence rates seen in the 65 to 74 age group was not as apparent in the 75 to 84 and $\geq 85$ age groups. Yet, during more than half of the years from 1973 to 2013, Black adults aged 75 to 84 and $\geq 85$ years had higher lung and bronchus cancer incidence rates than White adults.

Breast cancer-Similar to lung and bronchus cancer, breast cancer incidence increased over time among older White and Black women in the three age groups. In general, White 
women had higher breast cancer incidence rates than Black women. However, breast cancer incidence rates among older Black women neared the rates of their White counterparts within the past decade. Notably, Black women aged $\geq 85$ years had higher breast cancer incidence rates than White women in 2005-2008, 2012, and 2013.

Prostate cancer-Overall prostate cancer incidence declined over time among older White and Black men aged 75 to 84 and $\geq 85$ years, with the exception of the increase in the early 1990s after the advent of prostate-specific antigen (PSA) screening recommendations. For older White and Black men in the 65- to 74-year-old age group, prostate cancer incidence increased over time from 1973 to 2013. Older Black men in all three age groups had higher prostate cancer incidence rates than their White counterparts from 1973 to 2013.

\section{Stage at Diagnosis}

Earlier stage at diagnosis-Cancer stage at diagnosis among older men and women differed between Whites and Blacks. In general, White men and women had earlier stage at diagnosis (e.g., in situ, localized) for colorectal, lung and bronchus, breast, and prostate cancers compared with Black men and women aged 65 to 74,75 to 84 , and $\geq 85$ years. Specifically, White adults aged 65 to 74,75 to 84 , and $\geq 85$ years had higher proportions of localized colorectal $(40.3 \%, 39.8 \%$, and $34.5 \%$ vs. $37.5 \%, 36 \%$, and $30.5 \%)$, lung and bronchus $(20.8 \%, 21.4 \%$, and $16.9 \%$ vs. $17.2 \%, 18.2 \%$, and $16.3 \%)$, and breast (56.8\%, $58.7 \%$, and $55.1 \%$ vs. $48 \%, 49.9 \%$, and $48.1 \%$ ) cancers compared with Blacks, respectively.

Later stage at diagnosis-Black adults aged 65 to 74,75 to 84 , and $\geq 85$ years also had higher proportions of distant stage colorectal $(21.6 \%, 21.8 \%$, and $20.5 \%$ vs. $18 \%, 16.9 \%$, and $17 \%$ ), lung and bronchus $(54.6 \%, 53.4 \%$, and $50.8 \%$ vs. $51 \%, 49 \%$, and $49.1 \%$ ), and breast $(6 \%, 7.3 \%$, and $8.6 \%$ vs. $4.1 \%, 5 \%$, and $6.4 \%)$ cancers compared with Whites, respectively. Last, Blacks aged 65 to 74,75 to 84 , and $\geq 85$ years had higher proportions of unstaged $/$ missing stage colorectal $(4.9 \%, 7.4 \%$, and $18.5 \%$ vs. $3.6 \%, 5.5 \%$, and $14.3 \%)$, breast $(1.4 \%, 3 \%$, and $9.8 \%$ vs. $1.1 \%, 2 \%$, and $8.4 \%)$, and prostate $(3.5 \%, 9.4 \%$, and $33.5 \%$ vs. $2.9 \%, 7.1 \%$, and $24.8 \%$ ) cancers compared with Whites (Figure 3a-3d).

\section{Additional Tumor Characteristics and Treatment}

Table 1 shows the tumor and clinical characteristics of older White and Black adults by cancer type and age group. Older Black adults had larger median tumor sizes for lung and bronchus and breast cancers, and similar sizes for the colorectal cancer tumors for the 65 to 74 and $\geq 85$ age groups. Tumor sizes also increased as age increased in the sample. There were higher percentages of poorly differentiated/undifferentiated and unknown tumor grades for all cancer types, with the exception of colorectal, lung and bronchus, and prostate cancers among the $\geq 85$ age group.

Older Black adults less often received surgery for all four cancer types. Receipt of radiation was mixed; older Black adults received radiation at higher rates than older White adults for lung and bronchus, colorectal ( $\geq 85$ age group only), and prostate cancers (65-74 and $\geq 85$ age groups). On the contrary, older White adults received radiation at higher rates for breast, colorectal (65-74 and 75-84 age groups), lung and bronchus ( $\geq 85$ age group only), and 
prostate cancers (75-84 age group only). Last, receipt of surgery and radiation declined as age increased in all four cancer types.

\section{Survival}

The 5-year relative survival probability for all invasive cancers combined was lower for older Blacks than Whites- $54.5 \%$ (95\% confidence interval $[\mathrm{CI}]=[54.1,55.0])$ vs. $59.9 \%$ $(95 \% \mathrm{CI}=[59.8,60.1]) —$ not shown in figures. Specifically, older Black adults aged 65 to 74,75 to 84 , and $\geq 85$ years had lower 5-year relative survival with the exception of localized and regional prostate cancer in the 65- to 74- and 75- to 84-year-old age groups and unstaged/missing stage breast cancer in the 285 -year-old age group compared with Whites.

Table 2 shows that relative survival probabilities for colorectal cancer diagnosed at localized, regional, and distant stages were lower for Blacks aged 65 to 74,75 to 84 , and $\geq 85$ years than Whites, respectively. Blacks aged 65 to 74,75 to 84 , and $\geq 85$ years had lower relative survival probabilities of lung and bronchus cancer diagnosed at localized, regional, and distant stages than their White counterparts, respectively. Black women had lower relative survival probability of breast cancer diagnosed at localized, regional, and distant stages than White women, respectively. Last, Black men aged 65 to 74,75 to 84 , and $\geq 85$ years had lower relative survival probability of prostate cancer diagnosed at localized, regional, and distant stages than White men of the same age groups.

\section{Discussion}

This study sought to identify the presence of disparities in cancer incidence, stage at diagnosis, and survival between White and Black adults aged 65 to 74,75 to 84 , and $\geq 85$ years, regardless of ethnicity. Results demonstrated the continuation of White-Black differences across the cancer continuum among older adults in the United States. The most striking difference was that the 5-year relative survival probability for all invasive cancers was considerably higher for older White adults than older Black adults in all three age groups and increased with age. As seen in this study and the literature, differences in treatment, particularly reductions in recommended treatments (e.g., curative surgery, chemotherapy) and less aggressive treatments, contribute to White- Black differences in relative survival (Gross et al., 2008; Hardy et al., 2009). Ward and colleagues (2004) found that Black cancer patients were less likely to undergo recommended surgery than White cancer patients, even after controlling for socioeconomic factors. In addition, this study and the literature show that older patients are less likely to receive recommended treatments regardless of cancer stage (Foster et al., 2010; Freedman et al., 2009; Jessup et al., 2005; Mahal et al., 2014; Zeng et al., 2015). For example, Jessup and colleagues (2005) found that older adults have the same survival benefit from adjuvant chemotherapy as younger patients but are less frequently treated. In combination, older racial minorities may experience "double jeopardy" in that survival disparities are heightened when individuals are both a racial minority and older adult (Bellizzi et al., 2012).

J Aging Health. Author manuscript; available in PMC 2019 July 01. 


\section{Stage at Diagnosis}

Corresponding with previous research, this current study found that in each age group, older Black adults had later stages at diagnosis than their White counterparts (DeSantis et al., 2016; Mobley \& Kuo, 2016). Potential reasons for this disparity in stage at diagnosis are numerous, including SES, access to care, health literacy, and perception of cancer-related symptoms and beliefs about cancer (Lannin et al., 1998). Several studies (Bradley, Given, \& Roberts, 2002; Lannin et al., 1998; Lantz et al., 2006; Lipscomb et al., 2016; Maloney et al., 2006) have investigated whether racial disparities in stage at diagnosis remained once SES was taken into account. Results have been mixed. For example, Lannin and colleagues (1998; Lantz et al., 2006) found that racial differences in early-stage diagnosis remained despite controlling for SES, whereas Maloney et al. (2006) found the White-Black difference in stage at diagnosis was attenuated once SES was taken into account. A recent study by Lipscomb et al. (2016) found significant differences in stage at diagnosis among Blacks and Whites, but as the covariate set became richer, race became less influential. These findings raise important questions about the complex associations between race, SES, cultural beliefs, beliefs and attitudes about cancer screenings, geographic location, and health care utilization (Lantz et al., 2006). The lack of data on individual-level SES variables in population-based tumor registries presents an opportunity for social epidemiological research. Accounting for these multilevel and interconnected factors as seen in Warnecke et al.'s (2008) model for health disparities, researchers will be better able to understand the predictors of disparities in stage at diagnosis to target efforts to increase screening and improve delays in diagnostic follow-up.

\section{Cancer Screening and Incidence}

Cancer screening recommendations for older adults lack clarity and vary by organizations regarding when to stop screening. For example, U.S. Preventive Services Task Force (USPSTF) guidelines recommend against routine screening for breast, cervical, colorectal, and prostate cancer among adults aged $\geq 75$ years (USPSTF, 2016). On the contrary, the American Cancer Society does not have a limit to routine screenings and recommend "screening should continue as long as the individual in good health and is expected to live 10 more years or longer" (American Cancer Society, 2016). Despite these ambiguous recommendations, studies have found that older, racially diverse adults continue to be screened at high rates (Bellizzi, Breslau, Burness, \& Waldron, 2011; Marshall et al., 2016). Future research is needed to determine the benefits and harms to cancer screening among older adults to better inform screening guidelines. In addition, evidence-based decision tools addressing individual variations in life expectancy, functional status, comorbidities, and personal preference are needed to guide clinicians to objectively determine the appropriateness of screening among the diverse older adult population (Bellizzi et al., 2011; Salzman, Beldowski, \& de la Paz, 2016).

This study found, similar to other studies among all ages (DeSantis et al., 2016; Siegel et al., 2016), that in the 1970s and 1980s, colorectal incidence rates were generally higher in Whites than Blacks and beginning in the late 1980s and early 1990s, colorectal incidence rates have consistently been higher in Blacks than Whites. This crossover is possibly due to a combination of greater access to and utilization of colorectal cancer screening tests among

J Aging Health. Author manuscript; available in PMC 2019 July 01. 
Whites, as well as racial differences in trends for colorectal cancer risk factors (DeSantis et al., 2016; Irby, Anderson, Henson, \& Devesa, 2006). Although colorectal cancer screening rates have been increasing in both Whites and Blacks (DeSantis et al., 2016; National Center for Health Statistics) and most older adults are insured by Medicare which covers cancer screenings (Centers for Medicare \& Medicaid Services, 2016), disparities in colorectal cancer screening remain. According to the National Health Interview Survey (NHIS) 2010 data, $55.5 \%$ of Blacks received either a fecal occult blood test within the past year, sigmoidoscopy within the past 5 years, or a colonoscopy within the past 10 years, compared with $61.5 \%$ of Whites (National Center for Health Statistics).

\section{Strategies to Reduce Cancer Disparities}

One strategy to reduce this disparity and barriers to screening for colorectal and other cancers is through the use of community health workers (CHWs) and patient navigation (PN). CHWs work in the community to provide information, support, and encouragement to receive screening tests, and their role ends when the patient enters the health care facility (Viswanathan et al., 2010). PN is a patient-centered, health care service delivery model in clinics that assists individuals, particularly the medically underserved, in overcoming barriers to care (e.g., personal, logistical, system) across the cancer care continuum (Dohan \& Schrag, 2005). CHWs and patient navigators complement each other in that they serve as a bridge between underserved communities and the health care system. Previous studies have demonstrated both PN and CHW effectiveness in increasing knowledge (Ulloa et al., 2015; Viswanathan et al., 2010), improving rates of cancer screenings (Horne et al., 2015; Marshall et al., 2016), and ensuring follow-up rates after an abnormal screening test (Ko et al., 2016; Raich, Whitley, Thorland, Valverde, \& Fairclough, 2012) among racially diverse adults. However, PN and CHW interventions are limited in the older adult population (Horne et al., 2015; Marshall et al., 2016). Given the demographic changes among the cancer survivor population, examining the effectiveness of CHWs and PN among racially diverse older adults is warranted.

\section{Limitations}

There are several limitations of this study that should be considered. This study uses surveillance data that do not contain potentially confounding factors such as SES, access to care, physical functioning, and comorbidities. Additional resources such as the linked SEER-Medicare dataset would supply more information about the participants; however, the study's goal was to determine the presence of cancer disparities between older White and Black adults, and previous studies have shown cancer disparities between Whites and Blacks persist after controlling for these factors ( Du, Lin, Johnson, \& Altekruse, 2011; Kish, Yu, Percy-Laurry, \& Altekruse, 2014). Another limitation was that ethnicity was not examined in this study; thus, Whites and Blacks included Hispanics. Future research should consider including other racial groups and ethnic groups (e.g., Asian Americans, Hispanics/Latinos) who suffer disproportionally from cancer to better identify potential cancer disparities by race and ethnicity (Ward et al., 2004). It was not possible to examine changes in potential racial disparities and potentially similar confounding factors (e.g., by histology or SES) in mortality rates over time as race-, age-, sex-, and year-specific mortality rates are not easily available or routinely published. Last, as is common in research concerning survival 
probability, information about cancer diagnoses occurring in more recent years contributed less information to the calculation of relative survival probabilities; therefore, relative survival probabilities reported here may not fully characterize the impact of recent advances in treatment and additional factors related to survival.

\section{Conclusion}

This study found White-Black differences in cancer incidence rates, stage at diagnosis, and survival probabilities among older adults aged 65 to 74,75 to 84 , and -- $\geq 85$ years using SEER data. As previously determined in younger patient populations, the study demonstrated that racial disparities in four common cancer types exist in the older adult patient population. Better ways to reduce White-Black differences in cancer prevention and treatment among this population are urgently needed.

\section{Acknowledgments}

Funding

The authors received no financial support for the research, authorship, and/or publication of this article.

\section{References}

American Cancer Society. American Cancer Society guidelines for the early detection of cancer. 2016. Retrieved from http://www.cancer.org/healthy/findcancerearly/cancerscreeningguidelines/americancancer-society-guidelines-for-the-early-detection-of-cancer

Bellizzi KM, Aziz NM, Rowland JH, Weaver K, Arora NK, Hamilton AS, Keel G. Double jeopardy? Age, race, and HRQOL in older adults with cancer. Journal of Cancer Epidemiology 2012. 2012; Article ID 478642. doi: 10.1155/2012/478642

Bellizzi KM, Breslau ES, Burness A, Waldron W. Prevalence of cancer screening in older, racially diverse adults: Still screening after all these years. Archives of Internal Medicine. 2011; 171:20312037. DOI: 10.1001/archinternmed.2011.570 [PubMed: 22158573]

Bluethmann SM, Mariotto AB, Rowland JH. Anticipating the "silver tsunami": Prevalence trajectories and comorbidity burden among older cancer survivors in the United States. Cancer Epidemiology, Biomarkers \& Prevention. 2016; 25:1029-1036. DOI: 10.1158/1055-9965.epi-16-0133

Bradley CJ, Given CW, Roberts C. Race, socioeconomic status, and breast cancer treatment and survival. Journal of the National Cancer Institute. 2002; 94:490-496. [PubMed: 11929949]

Braveman P, Gottlieb L. The social determinants of health: It's time to consider the causes of the causes. Public Health Reports. 2014; 129(Suppl. 2):19-31.

Centers for Medicare \& Medicaid Services. Colorectal cancer screenings. 2016. Retrieved from https:// www.medicare.gov/coverage/colorectal-cancer-screenings.html

DeSantis CE, Siegel RL, Sauer AG, Miller KD, Fedewa SA, Alcaraz KI, Jemal A. Cancer statistics for African Americans, 2016: Progress and opportunities in reducing racial disparities. CA: A Cancer Journal for Clinicians. 2016; 66:290-308. DOI: 10.3322/caac.21340 [PubMed: 26910411]

Diez Roux AV. Conceptual approaches to the study of health disparities. Annual Reviews of Public Health. 2013; 33:41-58. DOI: 10.1146/annurev-publhealth-031811-124534

Dohan D, Schrag D. Using navigators to improve care of underserved patients: Current practices and approaches. Cancer. 2005; 104:848-855. DOI: 10.1002/cncr.21214 [PubMed: 16010658]

Du XL, Lin CC, Johnson NJ, Altekruse S. Effects of individual-level socioeconomic factors on racial disparities in cancer treatment and survival: Findings from the National Longitudinal Mortality Study, 1979-2003. Cancer. 2011; 117:3242-3251. DOI: 10.1002/cncr.25854 [PubMed: 21264829]

J Aging Health. Author manuscript; available in PMC 2019 July 01. 
Foster JA, Salinas GD, Mansell D, Williamson JC, Casebeer LL. How does older age influence oncologists' cancer management? Oncologist. 2010; 15:584-592. DOI: 10.1634/theoncologist. 2009-0198 [PubMed: 20495217]

Freedman RA, He Y, Winer EP, Keating NL. Trends in racial and age disparities in definitive local therapy of early-stage breast cancer. Journal of Clinical Oncology. 2009; 27:713-719. DOI: 10.1200/jco.2008.17.9234 [PubMed: 19103731]

Freeman HP, Chu KC. Determinants of cancer disparities: Barriers to cancer screening, diagnosis, and treatment. Surgical Oncology Clinics of North America. 2005; 14:655-669. [PubMed: 16226685]

Gross CP, Smith BD, Wolf E, Andersen M. Racial disparities in cancer therapy: Did the gap narrow between 1992 and 2002? Cancer. 2008; 112:900-908. DOI: 10.1002/cncr.23228 [PubMed: 18181101]

Hardy D, Liu CC, Xia R, Cormier JN, Chan W, White A, Du XL. Racial disparities and treatment trends in a large cohort of elderly black and white patients with nonsmall cell lung cancer. Cancer. 2009; 115:2199-2211. DOI: 10.1002/cncr.24248 [PubMed: 19365824]

Horne HN, Phelan-Emrick DF, Pollack CE, Markakis D, Wenzel J, Ahmed S, Ford JG. Effect of patient navigation on colorectal cancer screening in a community-based randomized controlled trial of urban African American adults. Cancer Causes \& Control. 2015; 26:239-246. DOI: 10.1007/s10552-014-0505-0 [PubMed: 25516073]

Howlader, N., Noone, AM., Krapcho, M., Garshell, J., Miller, D., Altekruse, SF., Cronin, KA. SEER cancer statistics review, 1975-2012. Bethesda, MD: National Cancer Institute; 2015. Retrieved from http://seer.cancer.gov/csr/1975_2012/

Irby K, Anderson WF, Henson DE, Devesa SS. Emerging and widening colorectal carcinoma disparities between Blacks and Whites in the United States (1975-2002). Cancer Epidemiology, Biomarkers \& Prevention. 2006; 15:792-797. DOI: 10.1158/1055-9965.epi-05-0879

Jessup JM, Stewart A, Greene FL, Minsky BD. Adjuvant chemotherapy for stage III colon cancer: Implications of race/ethnicity, age, and differentiation. Journal of the American Medical Association. 2005; 294:2703-2711. DOI: 10.1001/jama.294.21.2703 [PubMed: 16333005]

Kish JK, Yu M, Percy-Laurry A, Altekruse SF. Racial and ethnic disparities in cancer survival by neighborhood socioeconomic status in Surveillance, Epidemiology, and End Results (SEER) Registries. Journal of the National Cancer Institute Monographs. 2014; 2014:236-243. DOI: 10.1093/jncimonographs/lgu020 [PubMed: 25417237]

Ko NY, Snyder FR, Raich PC, Paskett ED, Dudley DJ, Lee JH, Freund KM. Racial and ethnic differences in patient navigation: Results from the Patient Navigation Research Program. Cancer. 2016; 122:2715-2722. DOI: 10.1002/cncr.30109 [PubMed: 27227342]

Lannin DR, Mathews HF, Mitchell J, Swanson MS, Swanson FH, Edwards MS. Influence of socioeconomic and cultural factors on racial differences in late-stage presentation of breast cancer. Journal of the American Medical Association. 1998; 279:1801-1807. [PubMed: 9628711]

Lantz PM, Mujahid M, Schwartz K, Janz NK, Fagerlin A, Salem B, Katz SJ. The influence of race, ethnicity, and individual socioeconomic factors on breast cancer stage at diagnosis. American Journal of Public Health. 2006; 96:2173-2178. DOI: 10.2105/ajph.2005.072132 [PubMed: 17077391]

25. Lipscomb J, Fleming ST, Trentham-Dietz A, Kimmick G, Wu XC, Morris CR, Sabatino SA. What predicts an advanced-stage diagnosis of breast cancer? Sorting out the influence of method of detection, access to care, and biologic factors. Cancer Epidemiology, Biomarkers \& Prevention. 2016; 25:613-623. DOI: 10.1158/1055-9965.epi-15-0225

Mahal BA, Ziehr DR, Aizer AA, Hyatt AS, Lago-Hernandez C, Choueiri TK, Nguyen PL. Racial disparities in an aging population: The relationship between age and race in the management of African American men with high-risk prostate cancer. Journal of Geriatric Oncology. 2014; 5:352358. DOI: 10.1016/j.jgo.2014.05.001 [PubMed: 24862107]

Maloney N, Koch M, Erb D, Schneider H, Goffman T, Elkins D, Laronga C. Impact of race on breast cancer in lower socioeconomic status women. The Breast Journal. 2006; 12:58-62. DOI: 10.1111/j.1075-122X.2006.00184.x [PubMed: 16409588]

Marshall JK, Mbah OM, Ford JG, Phelan-Emrick D, Ahmed S, Bone L, Pollack CE. Effect of patient navigation on breast cancer screening among African American Medicare beneficiaries: A

J Aging Health. Author manuscript; available in PMC 2019 July 01. 
randomized controlled trial. Journal of General Internal Medicine. 2016; 31:68-76. DOI: 10.1007/ s11606-015-3484-2 [PubMed: 26259762]

McKoy JM, Samaras AT, Bennett CL. Providing cancer care to a graying and diverse cancer population in the 21st century: Are we prepared? Journal of Clinical Oncology. 2009; 27:27452746. DOI: 10.1200/jco.2009.22.4352 [PubMed: 19403884]

Mobley LR, Kuo TM. Demographic disparities in late-stage diagnosis of breast and colorectal cancers across the USA. Journal of Racial and Ethnic Health Disparities. 2016; Advance online publication. doi: 10.1007/s40615-016-0219-y

National Center for Health Statistics. National Health Interview Survey 2000, 2013 and 2014. (Publicuse data file and documentation). Retrieved from cdc.gov/nchs/nhis.htm

Raich PC, Whitley EM, Thorland W, Valverde P, Fairclough D. Patient navigation improves cancer diagnostic resolution: An individually randomized clinical trial in an underserved population. Cancer Epidemiology, Biomarkers \& Prevention. 2012; 21:1629-1638. DOI: 10.1158/1055-9965.epi-12-0513

Salzman B, Beldowski K, de la Paz A. Cancer screening in older patients. American Family Physician. 2016; 93:659-667. [PubMed: 27175838]

Shanahan MJ, Hofer SM. Social context in gene-environment interactions: Retrospect and prospect. The Journals of Gerontology, Series B. Psychological Sciences \& Social Sciences. 2005; 60(Special Issue 1):65-76.

Siegel RL, Miller KD, Jemal A. Cancer statistics, 2016. CA: A Cancer Journal for Clinicians. 2016; 66:7-30. DOI: 10.3322/caac.21332 [PubMed: 26742998]

Smedley, BD.Stith, AY., Nelson, AR., editors. Unequal treatment: Confronting racial and ethnic disparities in health care. Washington, DC: National Academies Press; 2002.

Smith BD, Smith GL, Hurria A, Hortobagyi GN, Buchholz TA. Future of cancer incidence in the United States: Burdens upon an aging, changing nation. Journal of Clinical Oncology. 2009; 27:2758-2765. DOI: 10.1200/jco.2008.20.8983 [PubMed: 19403886]

Surveillance, Epidemiology, and End Results Program. SEER*Stat Database: Incidence-SEER 9 Regs Research Data, Nov 2015 Sub (1973-2013) <Katrina/Rita Population Adjustment>-Linked To County Attributes-Total U.S., 1969-2014 Counties. National Cancer Institute, Division of Cancer Control \& Population Sciences, Surveillance Research Program, Surveillance Systems Branch; 2016a. Apr. Available from www.seer.cancer.gov

Surveillance, Epidemiology, and End Results Program. SEER*Stat Database: Incidence-SEER 18 Regs Research Data + Hurricane Katrina Impacted Louisiana Cases, Nov 2015 Sub (1973-2013 varying)_Linked To County Attributes-Total U.S., 1969-2014 Counties. National Cancer Institute, Division of Cancer Control \& Population Sciences, Surveillance Research Program, Surveillance Systems Branch; 2016b. Apr. Available from www.seer.cancer.gov

Surveillance, Epidemiology, and End Results Program. Relative survival, cancer survival measures. 2017. Retrieved from https://seer.cancer.gov/seerstat/WebHelp/Relative_Survival.htm

Ulloa JG, Hemmelgarn M, Viveros L, Odele P, Feldman NR, Ganz PA, Maggard-Gibbons M. Improving breast cancer survivors' knowledge using a patient-centered intervention. Surgery. 2015; 158:669-675. DOI: 10.1016/j.surg.2015.03.056 [PubMed: 26032819]

U.S. Preventive Services Task Force. 2016. Published recommendations. Retrieved from http:// www.uspreventiveservicestaskforce.org/BrowseRec/Index

Viswanathan M, Kraschnewski JL, Nishikawa B, Morgan LC, Honeycutt AA, Thieda P, Jonas DE. Outcomes and costs of community health worker interventions: A systematic review. Medical Care. 2010; 48:792-808. DOI: 10.1097/MLR.0b013e3181e35b51 [PubMed: 20706166]

Wallace SP. Equity and social determinants of health among older adults. Generations. 2015; 38(4):611.

Ward E, Jemal A, Cokkinides V, Singh GK, Cardinez C, Ghafoor A, Thun M. Cancer disparities by race/ethnicity and socioeconomic status. CA: A Cancer Journal for Clinicians. 2004; 54:78-93. [PubMed: 15061598]

Warnecke RB, Oh A, Breen N, Gehlert S, Paskett E, Tucker KL, Hiatt RA. Approaching health disparities from a population perspective: The National Institutes of Health Centers for Population

J Aging Health. Author manuscript; available in PMC 2019 July 01. 
Health and Health Disparities. American Journal of Public Health. 2008; 98:1608-1615. DOI: 10.2105/ajph.2006.102525 [PubMed: 18633099]

Williams DR, Mohammed SA. Discrimination and racial disparities in health: Evidence and needed research. Journal of Behavioral Medicine. 2009; 32:20-47. [PubMed: 19030981]

Zeng C, Wen W, Morgans AK, Pao W, Shu XO, Zheng W. Disparities by race, age, and sex in the improvement of survival for major cancers: Results from the National Cancer Institute Surveillance, Epidemiology, and End Results (SEER) program in the United States, 1990 to 2010. Journal of the American Medical Association Oncology. 2015; 1:88-96. DOI: 10.1001/jamaoncol. 2014.161 


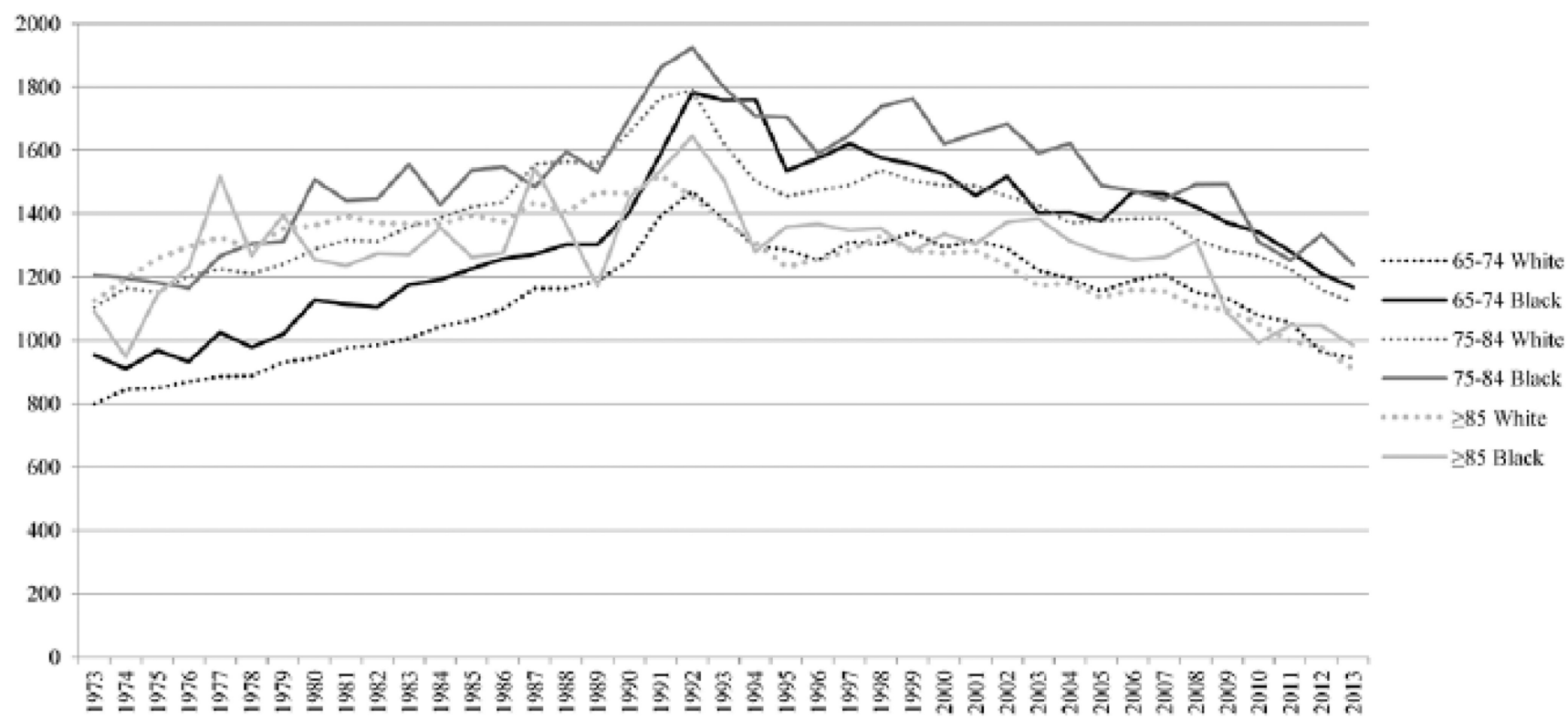

Figure 1.

Incidence Trends of Colorectal, Lung and Bronchus, Breast, and Prostate Cancer among Older White and Black Adults by Age and Race Group, 1973-2013. 
(a) Colorectal Cancer
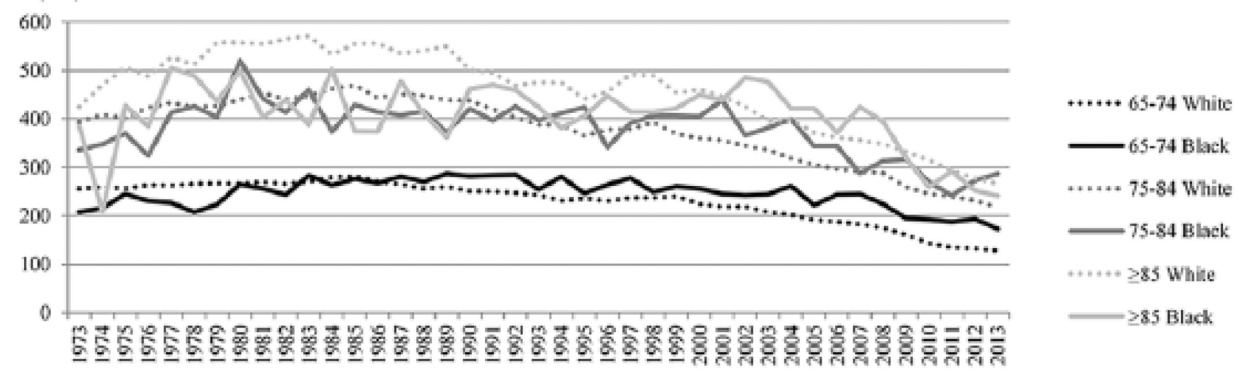

(b) Lung and Bronchus Cancer
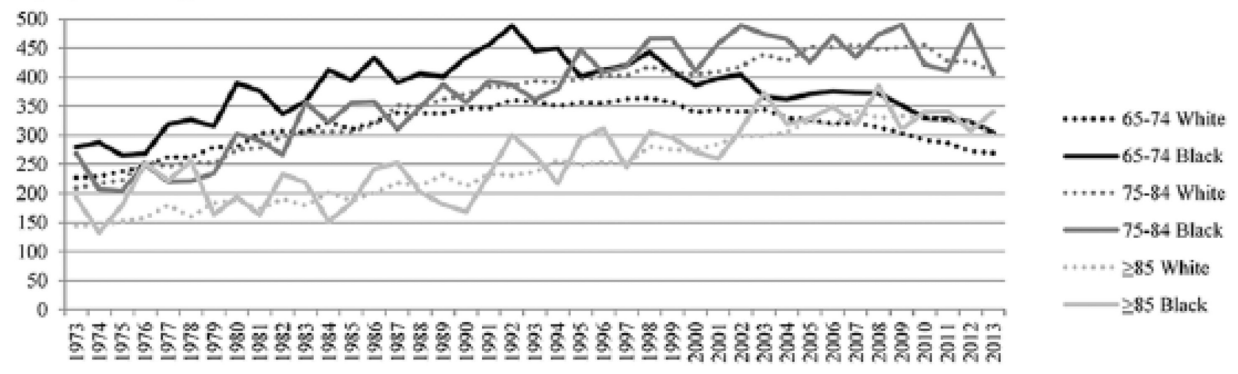

(c) Female Breast
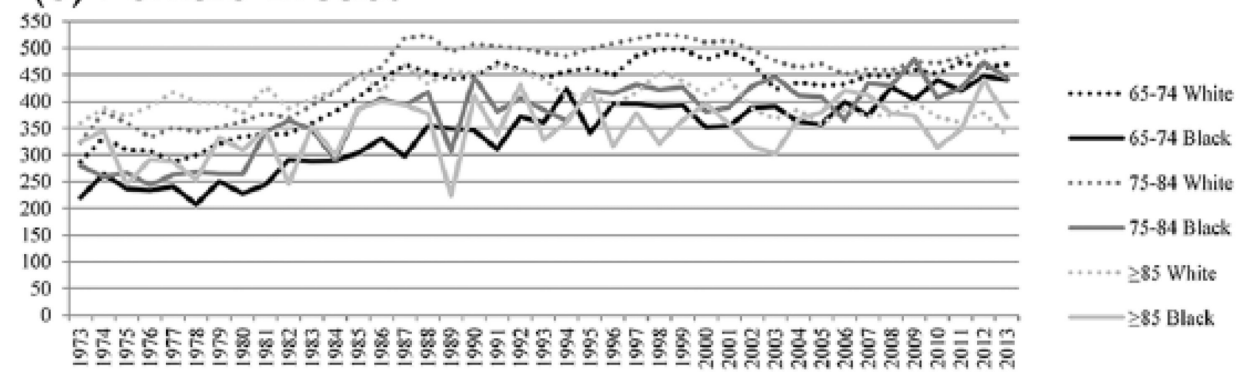

(d) Prostate Cancer
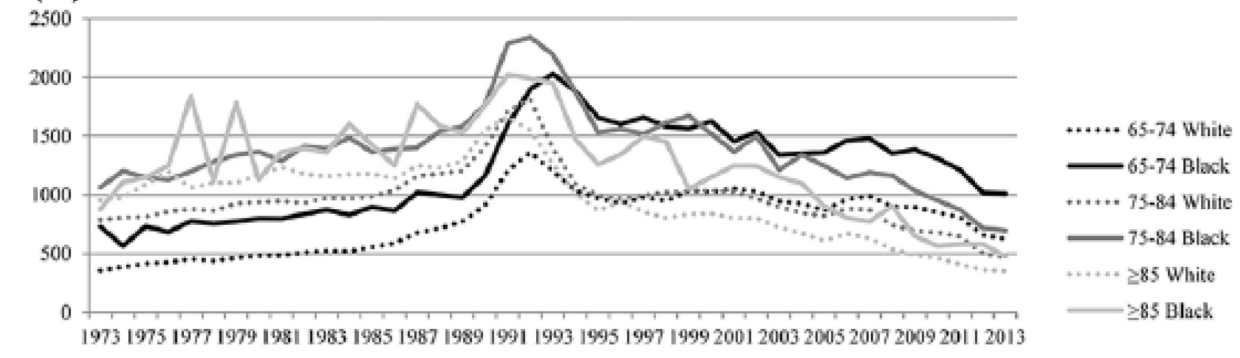

Figure 2.

Incidence Trends of Colorectal, Lung and Bronchus, Breast, and Prostate Cancer among Older White and Black Adults by Age and Race Group. 
(a) Colorectal Cancer

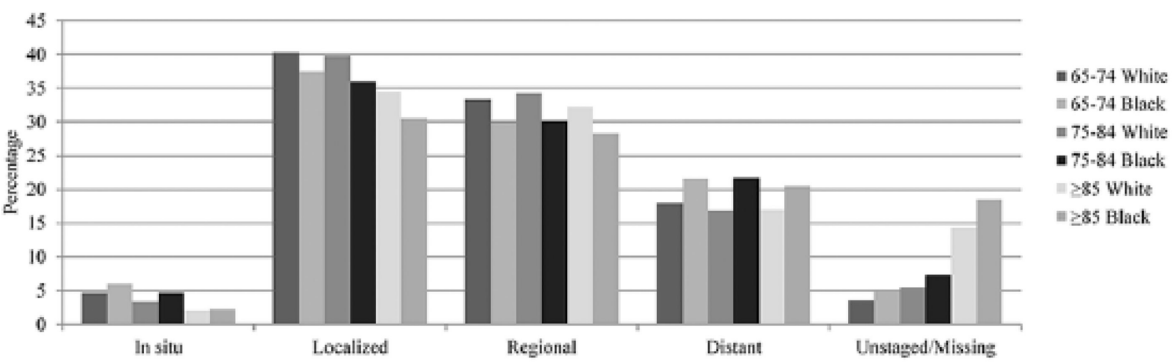

(b) Lung and Bronchus Cancer

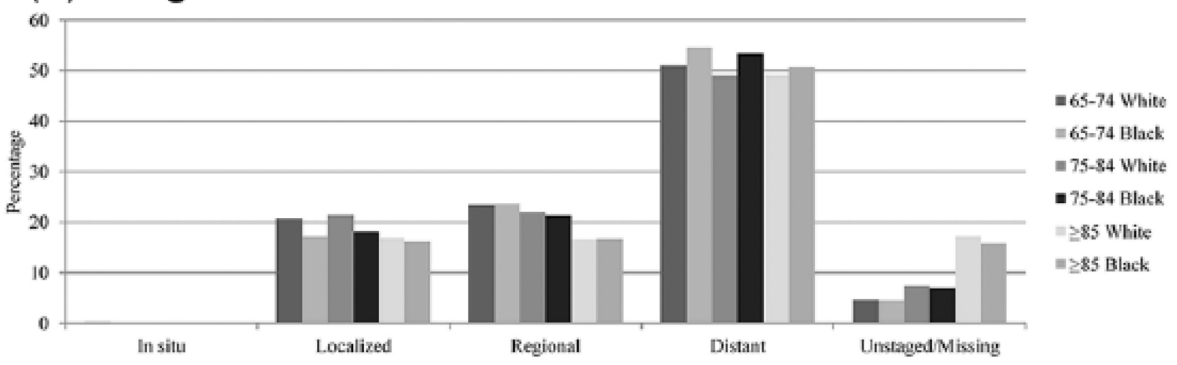

(c) Female Breast

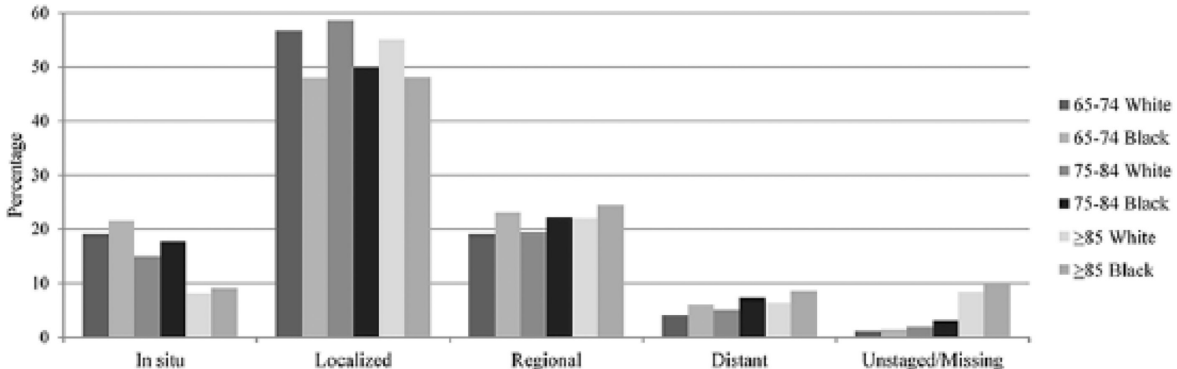

(d) Prostate Cancer

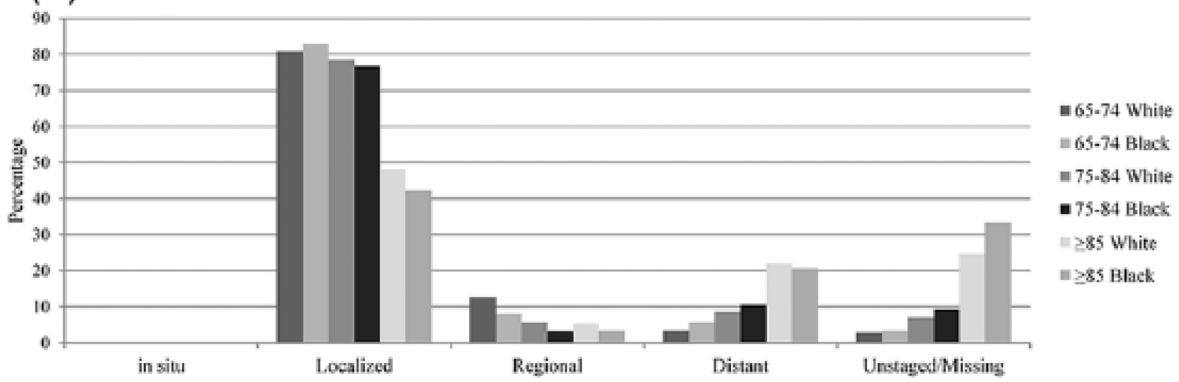

Figure 3.

a-d. Cancer Stage at Diagnosis among Older White and Black Adults by Age and Race Group, Diagnosed 2008-2012.

$J$ Aging Health. Author manuscript; available in PMC 2019 July 01. 


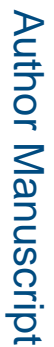

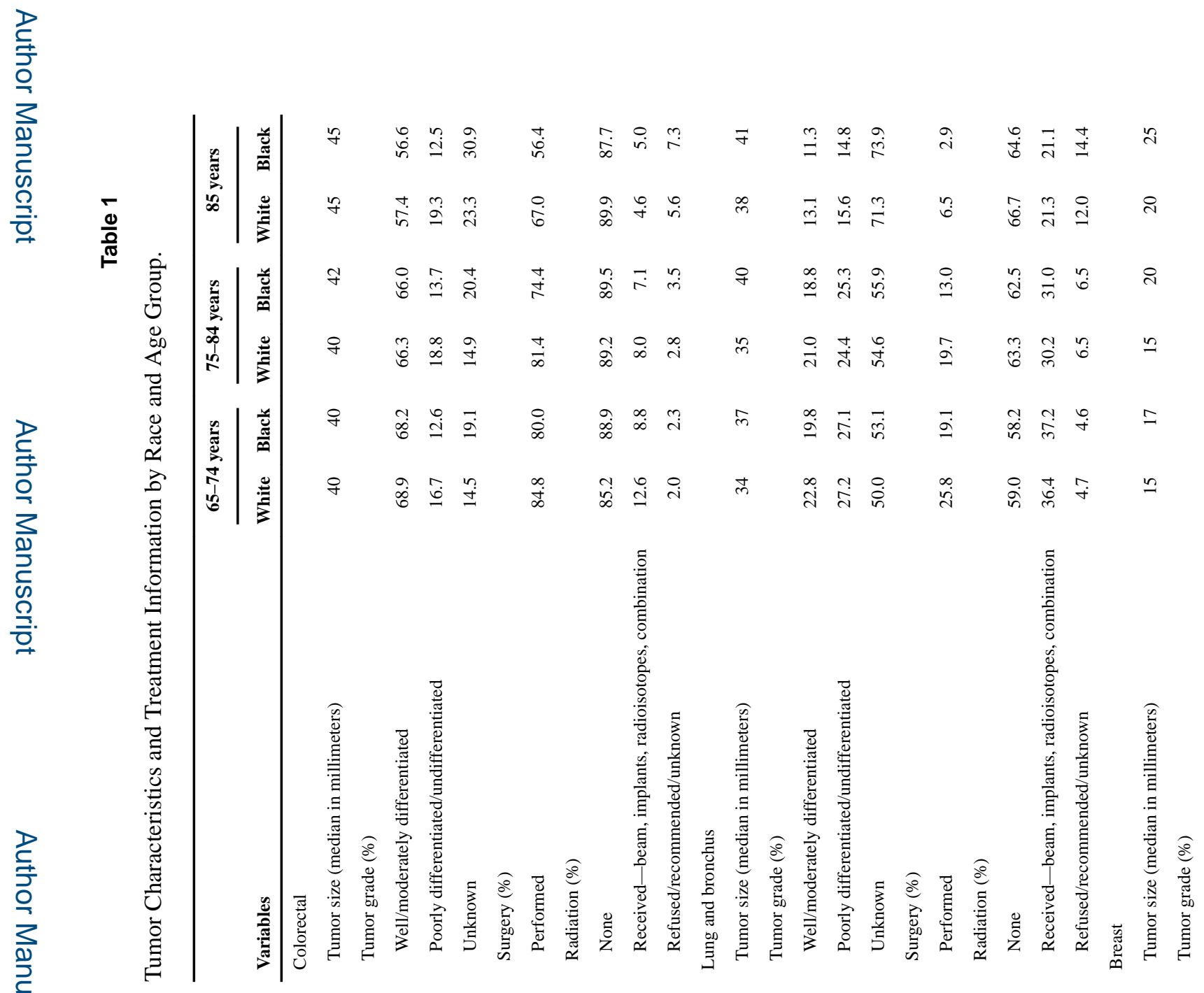




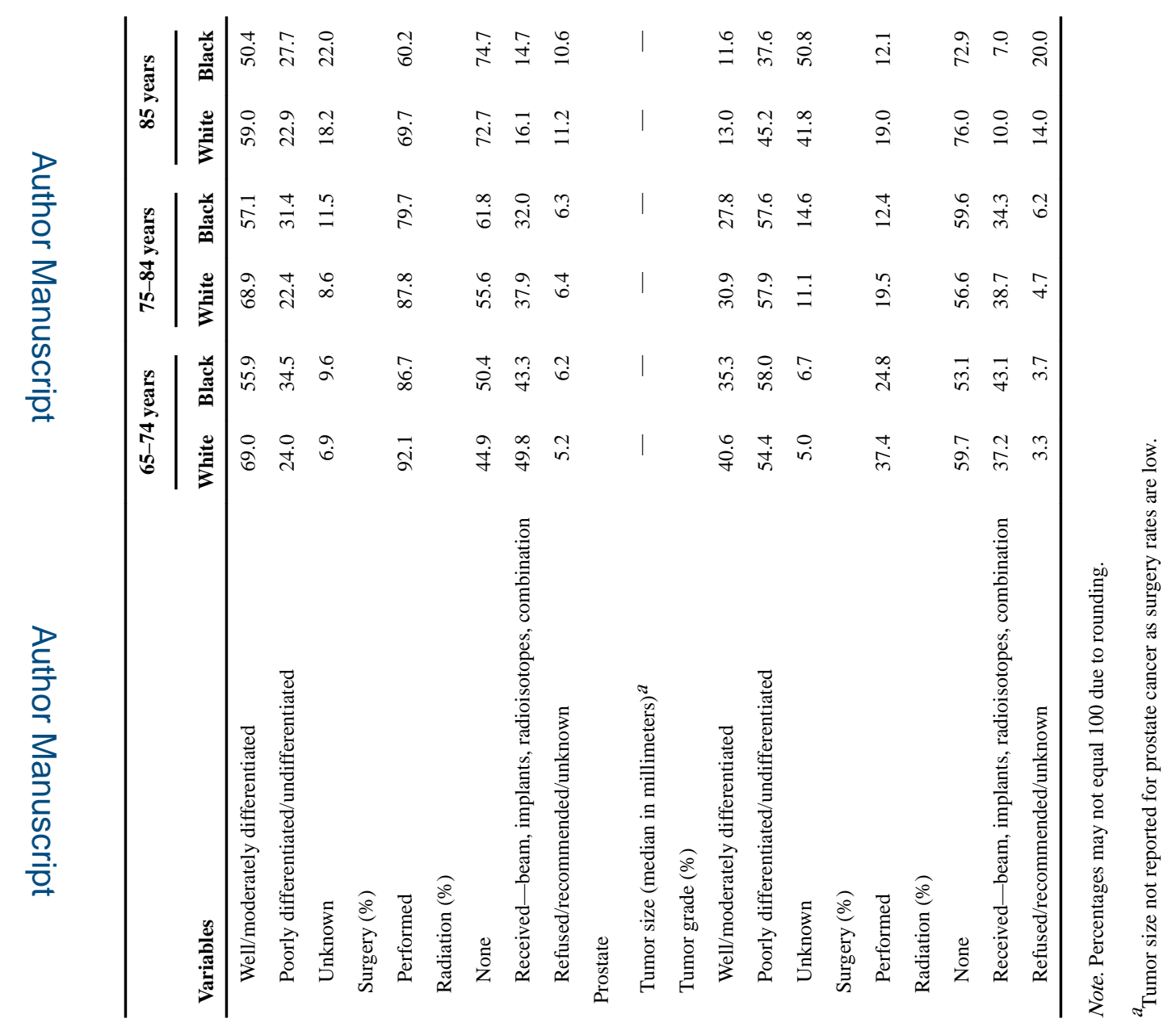

로을

로을

J Aging Health. Author manuscript; available in PMC 2019 July 01. 


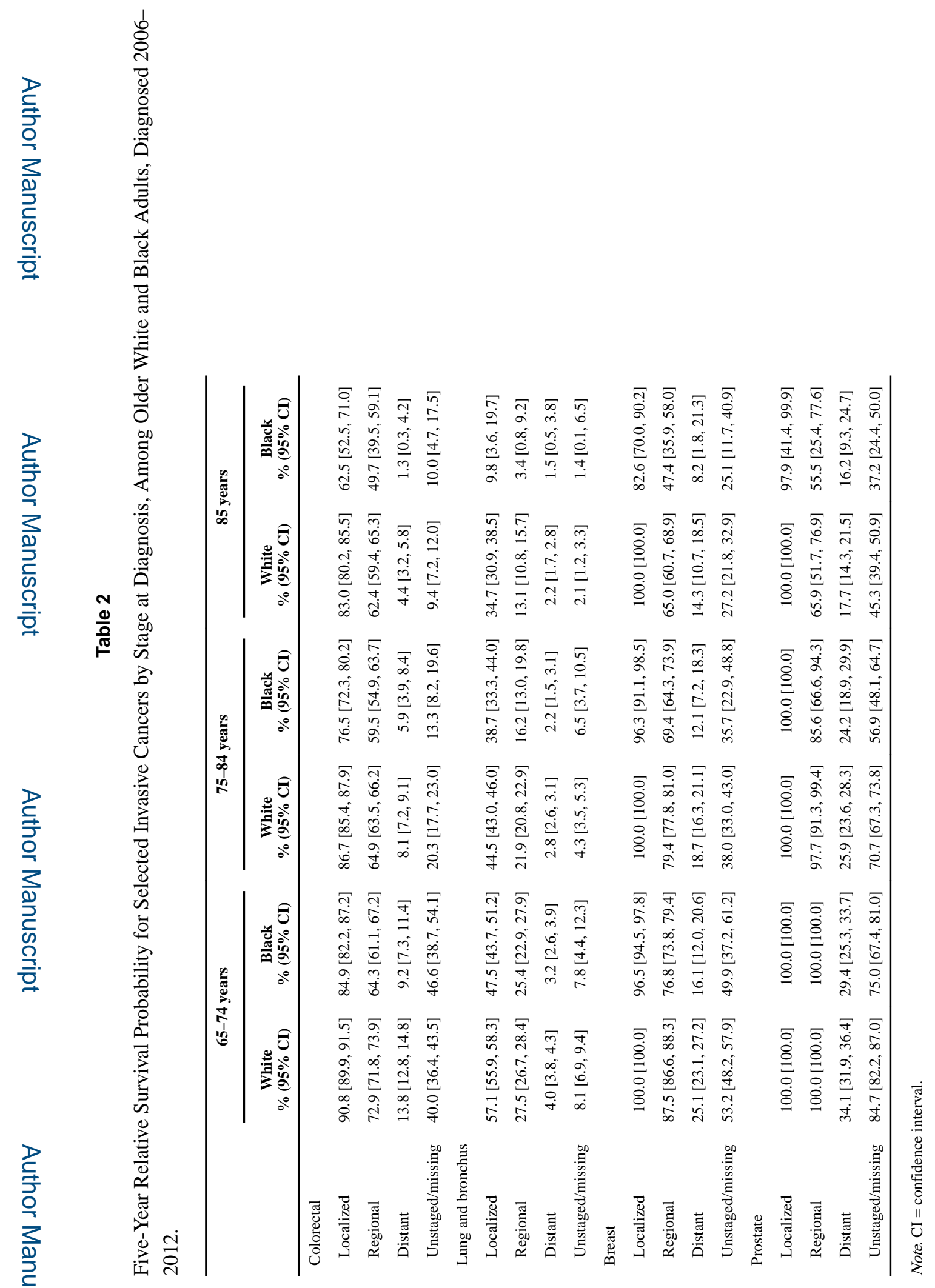

J Aging Health. Author manuscript; available in PMC 2019 July 01. 